\title{
FATORES QUE AFETAM A ADOÇÃO DA INTERNET MÓVEL
}

\section{FACTORS THAT AFFECT MOBILE INTERNET ACCEPTANCE}

Jorge Brantes Ferreira *

Professor Adjunto da Pontifícia Universidade Católica do Rio de Janeiro

Rio de Janeiro, RJ, Brasil

E-mail: jorgebf@gmail.com

Jorge Ferreira da Silva

Professor Titular da Pontifícia Universidade Católica do Rio de Janeiro

Rio de Janeiro, RJ, Brasil

E-mail: jorge1319@gmail.com

Cristiane Junqueira Giovannini

Douranda e Professora de Cursos de Extensão da Pontifícia Universidade Católica do Rio de Janeiro

Rio de Janeiro, RJ, Brasil

E-mail: mestrekis@gmail.com

Fernanda Leão Ramos

Professora de Cursos de Extensão da Pontifícia Universidade Católica do Rio de Janeiro

Rio de Janeiro, RJ, Brasil

E-mail: leaoramos@gmail.com

\section{RESUMO}

Esta pesquisa tem como objetivo investigar as percepções do consumidor ao avaliar a tecnologia de internet móvel, identificando os fatores que promovem a adoção deste serviço. É utilizada uma extensão do Modelo de Aceitação de Tecnologia (DAVIS, 1989), incorporando outros antecedentes que a literatura indica como relevantes para a avaliação do consumidor de serviços de internet móvel (como experiência com a internet, qualidade de conteúdo percebida, diversão percebida, qualidade do sistema percebida e nível de preço percebido). O teste das hipóteses foi feito utilizando equações estruturais com uma amostra de 305 consumidores. Os resultados destacam o papel da atitude como mediadora na explicação da intenção de uso da internet móvel. Além disso, a atitude foi notadamente mais influenciada pela utilidade percebida do que pela diversão percebida ou facilidade de uso percebida, com o nível de preço percebido não apresentando nenhum efeito significativo.

Palavras-chave: Internet Móvel. Aceitação de Tecnologias. Difusão de Inovações. Comportamento do Consumidor. Intenção de Uso.

Data de submissão: 19 de março de 2016.

\section{ABSTRACT}

This study aims to investigate consumers' perceptions regarding the adoption of mobile internet, identifying factors that might affect the use of this service. To evaluate this, an extension of the Technology Acceptance Model (DAVIS, 1989) is used, incorporating other antecedents that the literature indicates as relevant in the consumer's evaluation of mobile data services (such as internet experience, perceived content quality, perceived system quality, perceived playfulness and perceived price level). To test the proposed hypothesis, the study conducted a survey of 305 consumers, evaluating the data through structural equation modeling. The obtained results strengthen the notion that attitude fully mediates the relationship of the other constructs with the intention to use mobile internet services, with perceived usefulness presenting stronger effects on attitude than perceived ease of use or perceived playfulness, while perceived price level didn't present any significant effect.

Keywords: Mobile Internet. Technology Acceptance. Diffusion of Innovations. Consumer Behavior. Intention to Use.

Data de aprovação: 4 de setembro de 2016. 


\section{INTRODUÇÃO}

Globalmente, existem mais de sete bilhões de assinaturas de telefonia celular, indicando que serviços de telefonia móvel estão disponíveis para mais de 90\% da população mundial (IS, 2015). Só no Brasil, em junho de 2014, existiam 270 milhões de assinaturas de celular ativas (Anatel, 2015), e o processo de incrementar as redes de serviço a fim de que ofereçam a seus usuários acesso à internet tem ocorrido com mais velocidade que a adoção da telefonia celular propriamente dita. A ITU (International Telecommunications Union) indica uma penetração de $47 \%$ da banda larga móvel em escala global. Conexões $3 G$ estão disponíveis para $29 \%$ da população mundial em áreas rurais e $89 \%$ da população urbana (ITU, 2015). Além disso, segundo a ITU (2015), 95\% da população mundial tem acesso à internet 2G. Já existem mais pessoas acessando a internet por meio de conexões de banda larga móvel do que via conexões fixas.

A evolução das tecnologias oferecidas contribuiu muito para o crescimento da adoção da internet móvel. A tecnologia $3 G$ revolucionou o cenário da comunicação móvel, pois passou a permitir a transmissão de dados em alta velocidade. Como consequência, o $3 G$ possibilitou um maior acesso da população à internet. O Brasil possuía, ao fim de 2014, um volume de cerca de 145 milhões de acessos à rede 3G (ANATEL, 2015). O próximo passo é a difusão da tecnologia $4 \mathrm{G}$, que promete transformar o uso da internet móvel, com uma maior taxa de transferência de dados e melhor qualidade de serviço, e que contava em dezembro de 2014 com cerca de 6,7 milhões de acessos apenas no Brasil (ANATEL, 2015).

O dispositivo móvel, que já foi considerado um artigo de luxo, agora se tornou parte das ferramentas convencionais de comunicação. Os serviços móveis disponíveis, que antes ofereciam troca de dados de forma limitada, agora oferecem grande variedade de serviços, como e-mail, acesso ao sistema bancário, jogos, dentre outros. Com o crescimento do uso da internet, a maior penetração de dispositivos móveis e a rápida taxa de inovação tecnológica, a internet móvel representa uma grande oportunidade para negócios. Entretanto, apesar dos benefícios e flexibilidade oferecidos, na prática, há certa relutância na adoção da internet móvel. Então, a fim de promover seu uso, algumas condições devem ser atendidas. Primeiramente, é necessário que os dispositivos móveis sejam mais acessíveis à população e, em seguida, que o conteúdo fornecido ofereça serviços e informações úteis para os usuários. Por fim, é importante existam requisitos mínimos de rapidez, qualidade na comunicação e segurança para a promoção dessa tecnologia.

Estima-se que, em 2016, mais de dois bilhões de dólares em transações de comércio online serão realizadas através de aplicativos móveis no mundo inteiro, e que só nos Estados Unidos, $50 \%$ do lucro do comércio online virá de transações realizadas por meio de dispositivos móveis (GARTNER, 2014). No Brasil, em 2014, embora apenas 9,7\% das transações do comercio online tenham sido feitas por meio de dispositivos móveis sem o uso de apps (aplicativos específicos), o share do comércio móvel das operações de comércio online dobrou em relação ao ano anterior (E-BIT, 2015). E a previsão é que o faturamento do comércio online para 2015 seja em torno dos 43 bilhões de reais, um crescimento de 20\% em relação a 2014 (E-BIT, 2015). Dado este cenário, se torna necessária uma compreensão mais profunda dos interesses dos consumidores e a identificação dos fatores que apresentam maior influência sobre a adoção da internet móvel.

O objetivo deste artigo é o de analisar fatores envolvidos na intenção comportamental de uso da internet móvel pelo consumidor. Para tanto, empregou-se uma versão modificada do Modelo de Aceitação de Tecnologia (TAM - Technology Acceptance Model) de Davis (1989), com base em uma proposta de Cheong 
e Park (2005), que retrata de forma mais adequada o contexto da internet móvel. Com o intuito de buscar uma melhor compreensão da aceitação do consumidor em relação a essa tecnologia, foram utilizados cinco outros construtos, além daqueles já presentes no modelo TAM. Os novos construtos (diversão, qualidade do sistema, experiência com a internet, qualidade do conteúdo e nível de preço percebido) abordam particularidades da tecnologia avaliada e retratam características relevantes para o entendimento da adoção da internet móvel (CHEONG; PARK, 2005).

\section{REVISÃO DA LITERATURA}

\section{Aceitação de Tecnologia}

O uso e adoção de sistemas de informação nas organizações é o tema central de muitas pesquisas devido à sua importância para a difusão de tecnologias. Na última década, diversos estudos obtiveram resultados relevantes na análise da aceitação de usuários com relação a sistemas de informação em geral (DAVIS et al., 1989; YOUSAFZAI et al., 2007; PAI; HUANG, 2011; CHEUNG; VOGEL, 2013) e internet móvel em particular (CHEONG; PARK, 2005; BRUNER; KUMAR 2005; WANG; WANG, 2010; GERPOTT, 2011; KOENIGSTORFER; GROEPPEL-KLEIN, 2012).

O Modelo de Aceitação de Tecnologia (TAM) de Davis (1989), originalmente desenvolvido para explicar a aceitação de tecnologias da informação, foi elaborado com base na Teoria da Ação Racionalizada (TRA) concebida por Fishbein e Ajzen (1975) e posteriormente expandido para incluir outras tecnologias e serviços, como o comércio móvel (GERPOTT, 2011). A TRA sugere que o comportamento humano está diretamente associado à intenção, que por sua vez é baseada nas crenças de um indivíduo sobre aquele comportamento. Davis (1989) define a aceitação de tecnologia como a intenção voluntária de empregar uma tecnologia seguida posteriormente da adoção e uso real desta e utiliza construtos cognitivos (utilidade percebida e facilidade de uso percebida) como antecedentes da atitude em relação à adoção desta tecnologia. A utilidade percebida se refere à extensão na qual um indivíduo acredita que o seu desempenho irá melhorar ao utilizar uma tecnologia. Já a facilidade de uso percebida é definida pela extensão na qual um indivíduo acredita que o uso de um determinado sistema será desprovido de dificuldade ou esforço.

O TAM é um dos modelos mais influentes e discutidos para explicar o comportamento do usuário final e o uso de um sistema (YOUSAFZAI et al., 2007; HSIAO; YANG, 2011). No entanto, dependendo do contexto de cada tecnologia específica, outras explicações adicionais são necessárias, além dos construtos iniciais (Davis, 1989). Fatores que contribuem para a aceitação de novas tecnologias da informação variam de acordo com a tecnologia, o público-alvo e o contexto (DAVIS, 1989; GEFEN et al., 2003; CHEONG; PARK, 2005; BRUNER; KUMAR, 2005; GERPOTT, 2011; HSIAO; YANG, 2011; AKTURAN; TEZCAN, 2012; NASRI; CHARFEDDINE, 2012; TSENG; TENG, 2014; FERREIRA et al., 2014).

Pesquisadores têm obtido sucesso adotando e adaptando o Modelo de Aceitação de Tecnologia para a adoção de novas tecnologias relacionadas ao uso da internet e do e-commerce (MOON; KIM, 2001; GEFEN et al., 2003; HSIAO; YANG, 2011; NASRI; CHARFEDDINE, 2012; TSENG; TENG, 2014; WU; KE, 2015).

Para analisar o contexto de internet em dispositivos móveis, Cheong e Park (2005) estenderam o modelo TAM empregando cinco outros construtos, com o objetivo de melhor explicar a adoção e utilização da 
internet móvel: a diversão percebida, a experiência na internet, a qualidade do conteúdo, a qualidade do sistema e o nível de preço percebido.

A Figura 1 apresenta o modelo testado neste estudo, segundo proposto por Cheong e Park (2005), juntamente com suas hipóteses.

Figura 1. Modelo e hipóteses testadas (Cheong \& Park, 2005).

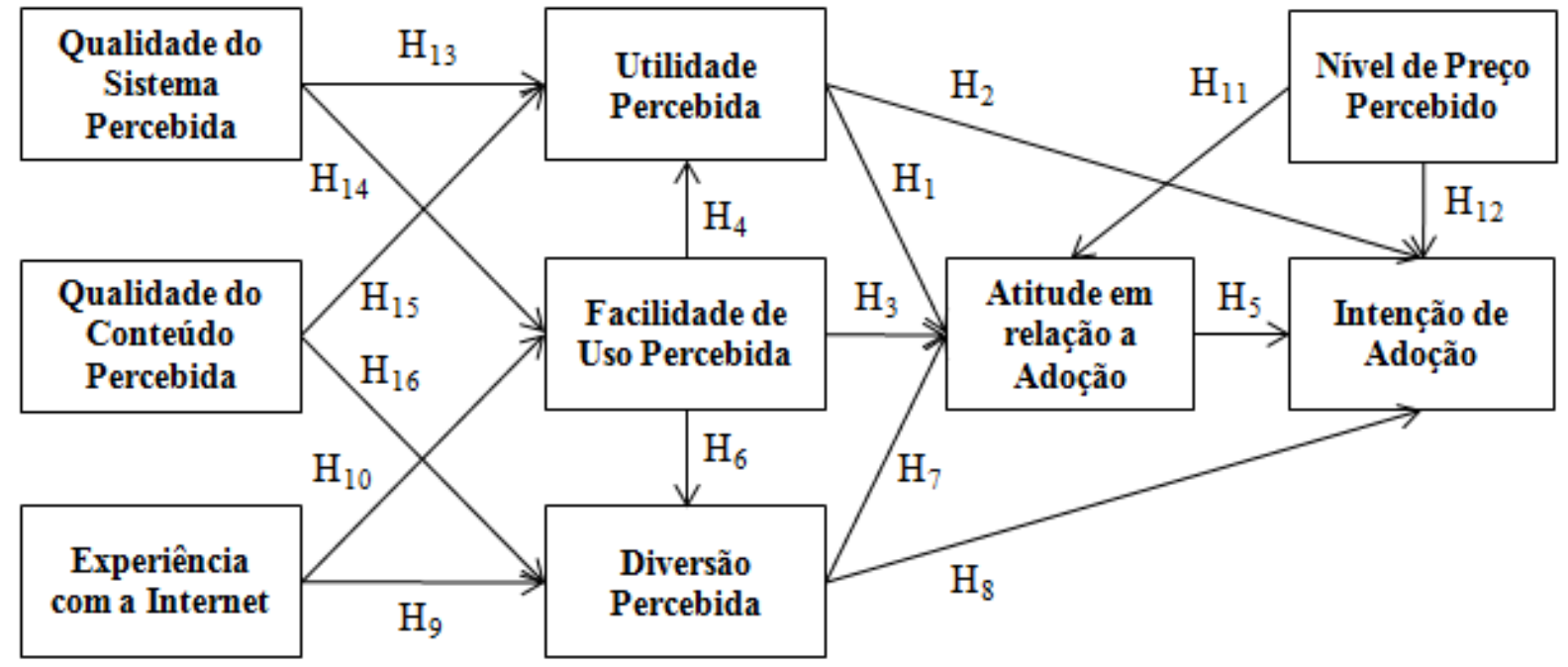

Fonte: elaborada pelos autores.

\section{Utilidade Percebida}

Há uma série de pesquisas em relação aos sistemas de informação que apresentam evidências de que a utilidade percebida possui influência na intenção de uso (SCHULTZ; SLEVIN, 1975; ROBEY, 1979; DAVIS et al., 1989; VENKATESH; DAVIS, 2000).

No caso do consumidor, a utilidade percebida está atrelada ao resultado funcional do uso da tecnologia, podendo ser traduzida como a probabilidade, percebida pelo consumidor, de que a tecnologia em questão irá beneficiá-lo de alguma forma ao realizar uma tarefa. Relações positivas e significativas foram encontradas entre a utilidade percebida e a atitude com relação ao uso de novas tecnologias. Alguns exemplos são o uso de novos serviços disponíveis na internet (CHILDERS et al., 2001; GENTRY; CALANTONE, 2002; NASRI; CHARFEDDINE, 2012; TSENG; TENG, 2014; WU; KE, 2015) e a atitude em relação a utilizar produtos que permitam o acesso móvel à Internet (BRUNER; KUMAR, 2005; AKTURAN; TEZCAN, 2012; WU; KE, 2015). Espera-se, portanto, que a utilidade percebida por consumidores sobre o uso da internet móvel tenha efeitos positivos diretos sobre sua atitude e intenção de uso desta tecnologia.

Hipótese 1: A utilidade percebida pelo consumidor terá um efeito positivo direto sobre a atitude em relação à internet móvel.

Hipótese 2: A utilidade percebida pelo consumidor terá um efeito positivo direto sobre a sua intenção de usar a internet móvel. 


\section{Facilidade de Uso Percebida}

O construto facilidade de uso percebida envolve a crença do indivíduo sobre o quão fácil ou livre de esforço é a tarefa de aprender a utilizar (e posteriormente usar) uma tecnologia. A literatura apresenta evidências significativas do efeito da facilidade de uso percebida sobre a atitude e intenção de adoção; tanto de forma direta quanto indireta, mediada pela utilidade percebida (WANG et al., 2006; GERPOFF, 2011; AKTURAN; TEZCAN, 2012; NASRI; CHARFEDDINE, 2012; TSENG; TENG, 2014; WU; KE, 2015). No entanto, Venkatesh e Davis (2000) ressaltam que os efeitos da facilidade de uso percebida sobre a intenção de uso de uma determinada tecnologia diminuem à medida que os usuários se tornam mais familiarizados com o sistema, ou em casos em que a complexidade do sistema é muito baixa.

No contexto da internet móvel, quanto maior for a percepção dos usuários em relação à facilidade e simplicidade do uso do serviço, maior será o nível de eficiência percebido. Por outro lado, a facilidade de uso ajuda a melhorar as percepções dos usuários em relação aos serviços oferecidos pela internet móvel, portanto, tornando-os mais dispostos a perceber utilidade em seu uso. Dessa forma, o website mobile deve ser fácil de entender, de buscar informações e principalmente fácil de comprar (HAILIN, 2010). Sendo assim, elaboram-se as seguintes hipóteses:

Hipótese 3: A facilidade de uso percebida pelo consumidor terá um efeito positivo direto sobre a sua atitude em relação à internet móvel.

Hipótese 4: A facilidade de uso percebida pelo consumidor terá um efeito positivo direto sobre a utilidade percebida.

\section{Atitude com relação à adoção}

O papel da atitude como mediador da intenção de adoção é razão de debate na literatura sobre aceitação de tecnologia. Sheppard et al. (1988), em uma meta-análise da teoria da ação racionalizada, concluíram que a atitude estaria significativamente relacionada com a intenção de uso. Se por um lado, Venkatesh (1999) e Venkatesh e Davis (2000), estudando o papel da atitude no modelo TAM, apontam para dúvidas sobre o seu papel como construto mediador dos efeitos da utilidade percebida e da facilidade de uso sobre a intenção. Por outro lado, Bruner e Kumar (2005) comentam que, no modelo c-TAM (consumer Technology Acceptance Model), proposto por eles, não foram vistos efeitos diretos do construto utilidade percebida sobre a intenção de adoção, somente tendo sido verificados efeitos indiretos via atitude. Seja de maneira direta ou indireta, a atitude desempenharia, portanto, papel importante na aceitação de tecnologia por consumidores. Desta forma, propõe-se:

Hipótese 5: A atitude do consumidor em relação à internet móvel terá um efeito positivo direto sobre a intenção de uso desta tecnologia. 


\section{Diversão Percebida}

Atualmente, a internet é utilizada tanto para fins profissionais, quanto como forma de entretenimento (CHEONG; PARK, 2005; OH et al., 2009). Assim, diversos estudos enfatizam a importância de incluir um construto relacionado ao entretenimento para melhor entender a intenção dos indivíduos nesse contexto (MOON; KIM, 2001; HEIJDEN, 2003; NYSVEEN et al., 2005; FANG et al., 2006; OH et al., 2009; WANG; WANG, 2010; WU; KE, 2015). O conceito de diversão percebida desempenharia um papel crucial na adoção de novas tecnologias, explicando tanto a atitude, quanto a intenção do indivíduo de navegar, interagir e comprar online (CHEONG; PARK, 2005; TAN; CHOU, 2008; OH et al., 2009; ÇELIK, 2011; WU; KE, 2015).

A diversão percebida é definida como a extensão na qual um indivíduo acredita que usar um produto ou serviço é agradável por si só, à parte de qualquer consequência desse uso que possa ser antecipada (MOON; KIM, 2001).

É lógico supor que sistemas mais simples de usar serão também potencialmente mais divertidos (AGARWAL; KARAHANNA, 2000; CHEONG; PARK, 2005; WANG; LIN, 2012). Dessa forma, quanto maior a percepção do consumidor da facilidade de uso da internet móvel, mais provável será que ele considere essa atividade divertida e, posteriormente, adote essa tecnologia (MOON; KIM, 2001; HEIJDEN, 2003; CHUNG; TAN, 2004; WANG; LIN, 2012).

Com base nesses argumentos, as seguintes hipóteses são propostas:

Hipótese 6: A facilidade de uso percebida pelo consumidor terá um efeito positivo direto sobre a diversão percebida por ele ao utilizar a internet móvel.

Hipótese 7: A diversão percebida pelo consumidor terá um efeito positivo direto sobre a sua atitude em relação à internet móvel.

Hipótese 8: A diversão percebida pelo consumidor terá um efeito positivo direto sobre a sua intenção de usar a internet móvel.

\section{Experiência com a Internet}

É comum que em suas interações iniciais com uma tecnologia, as pessoas se sintam inseguras, entretanto, conforme ganham experiência em seu uso, familiarizando-se com seus processos, é possível que desenvolvam percepções mais favoráveis em relação à dificuldade de uso da tecnologia, sua utilidade e sua capacidade de entreter (HACKBARTH et al., 2003; CHEONG; PARK, 2005; LIN, 2012; AL-QEISI et al., 2014). De fato, as pessoas tendem a adotar tecnologias que são similares aquelas previamente utilizadas (CHEONG; PARK, 2005; NASERI; ELLIOT, 2011; TSAI; LAROSE, 2015).

Em sua Teoria de Difusão de Inovações, Rogers (2003), sugere que as características das inovações e como elas são percebidas pelos indivíduos influenciam em sua adoção e difusão. Segundo Rogers (2003), a compatibilidade de uma inovação, definida como o grau com o qual a inovação é percebida como sendo consistente com valores já existentes, experiências passadas e as necessidades dos potenciais adotantes, é uma dessas características. Assim, as pessoas teriam uma maior tendência a adotar uma nova tecnologia 
quando esta Ihes é familiar ou compatível com outras tecnologias e sistemas que já conhecem (ROGERS, 2003; LU; SU, 2009; NASERI; ELLIOT, 2011; TSAI; LAROSE, 2015).

Diversos estudos sobre comportamento online e aceitação de tecnologia indicam que experiência previa com a internet e o conhecimento das tecnologias envolvidas exercem efeitos diretos e significativos sobre as percepções, influenciando expectativas, atitudes e intenções (CHEONG; PARK, 2005; NASERI; ELLIOT, 2011; VAN DEURSEN; VAN DIJK; PETERS, 2011; TOUFAILY et al., 2013; AL-QEISI et al., 2014; TSAI; LAROSE, 2015).

Dada a literatura, foram desenvolvidas as seguintes hipóteses:

Hipótese 9: A experiência com a internet do consumidor tem um efeito positivo direto sobre diversão percebida por ele ao utilizar a internet móvel.

Hipótese 10: A experiência com a internet do consumidor terá um efeito positivo direto sobre a facilidade de uso percebida por ele ao utilizar a internet móvel.

\section{Nível de Preço Percebido}

Durante a formação da intenção comportamental, os consumidores comparam custos e benefícios do uso de um determinado serviço (CHEONG; PARK, 2005; WANG; WANG, 2010; GERPOTT, 2011; MONROE, 2012). Se os benefícios de um serviço forem insuficientes para compensar seus custos, os consumidores não estarão dispostos a pagar por ele. (ZEITHAML, 1988; MONROE, 2012).

O custo (ou preço) pode ser definido como o que foi abandonado ou sacrificado para se obter um produto, seja este custo de ordem monetária, psicológica, cognitiva ou social (SHIN, 2010; MONROE, 2012). Isso torna o custo ou preço uma avaliação subjetiva, que representa o quanto os indivíduos estão dispostos a sacrificar em relação ao valor percebido do serviço oferecido, e um importante influenciador da intenção de uso de um serviço ou adoção de uma tecnologia (CHEONG; PARK, 2005; WANG; WANG, 2010, YOON et al., 2014).

No caso da internet móvel, o custo relacionado ao seu uso pode ser separado em dois componentes: o custo de aquisição do dispositivo móvel e a assinatura. Neste estudo optou-se por eliminar o custo de aquisição, pois este processo decisório envolve outras questões além da capacidade do dispositivo de acessar a Internet. Assim, foram elaboradas as seguintes hipóteses:

Hipótese 11: O nível de preço percebido pelo consumidor terá um efeito negativo direto sobre sua atitude em relação à internet móvel.

Hipótese 12: O nível de preço percebido pelo consumidor terá um efeito negativo direto sobre a sua intenção de usar a internet móvel. 


\section{Qualidade do Sistema}

Uma vez que demoras na resposta, falhas na conexão, indisponibilidade do acesso e baixos níveis de segurança podem provocar aversão à utilização de um sistema, a qualidade deste se configura como uma importante característica no caso de sistemas de informação (CHEN; CORKINDALE, 2008, KIM; HWANG, 2012; LIN; CHEN, 2012; AL-QEISI et al., 2014).

Estudos indicam que a qualidade da informação e do sistema tem papel fundamental na adoção de sistemas de informação (DELONE; MCLEAN, 1992, 2003; AL-QEISI et al., 2014) e na formação das percepções de sua utilidade e complexidade (LIN; LU, 2000; CHEN; CORKINDALE, 2008; LIN; CHEN, 2012; AL-QEISI et al., 2014). Ahn et al. (2007) sugerem que a qualidade do sistema também possui um impacto significativo sobre diversão percebida. Tempo de resposta, acessibilidade, estabilidade e segurança podem ser considerados como atributos que compõem o construto qualidade de um sistema de informação (AHN et al., 2007; LIN \& CHEN, 2012; AL-QEISI et al., 2014). Com esta base, foram definidas as seguintes hipóteses:

Hipótese 13: A qualidade do sistema percebida pelo consumidor terá um efeito positivo direto sobre a utilidade percebida por ele.

Hipótese 14: A qualidade do sistema percebida pelo consumidor terá um efeito positivo direto sobre a facilidade de uso percebida por ele.

\section{Qualidade do Conteúdo}

A qualidade do conteúdo vem sendo identificada como um dos fatores que mais afetam o uso da tecnologia móvel. Lin e Lu (2000) descobriram que a qualidade da informação oferecida por um website é um fator que afeta a percepção de sua utilidade. Para Cheong e Park (2005), conteúdo relevante pode fazer com que indivíduos achem a internet móvel mais agradável e divertida. Portanto, a relevância do conteúdo e a eficiência com que essas informações são exibidas podem ser consideradas atributos que compõem a qualidade do conteúdo. Ahn et al. (2007), por sua vez, sugerem que o impacto da qualidade do conteúdo sobre diversão percebida é mediada pela facilidade de uso percebida.

Para Pagani (2011), dado o tamanho reduzido dos dispositivos móveis, o conteúdo definitivamente terá uma grande relevância. Em seu estudo sobre serviços de TV móvel, a relevância do conteúdo foi considerada como o indicador mais importante em prever a atitude em relação à adoção. Além disso, o estudo sugere que a qualidade do conteúdo influencia significativamente o construto diversão percebida. Desta forma, propõem-se as seguintes hipóteses:

Hipótese 15: A qualidade do conteúdo percebida pelo consumidor terá um efeito positivo e direto sobre a utilidade percebida por ele.

Hipótese 16: A qualidade do conteúdo percebida pelo consumidor terá efeito positivo e direto sobre a facilidade de uso percebida por ele. 


\section{METODOLOGIA}

Com o objetivo de realizar o teste das hipóteses propostas foi realizado um cross-sectional survey (PARASURAMAN et al., 2006) com uma amostra não probabilística da população de interesse. Grande parte dos estudos sobre aceitação de tecnologia por consumidores utilizam esta mesma forma de pesquisa (CHILDERS et al., 2001; YOUSAFZAI et al., 2007; KULVIWAT et al., 2007; NASCO et al., 2008), com questionários estruturados sendo apresentados a consumidores em um único momento no tempo.

\section{Operacionalização das variáveis}

O estudo faz uso de escalas já elaboradas e testadas na literatura para a medição de todos os construtos envolvidos no modelo proposto. Sendo assim, foram utilizadas as seguintes escalas:

- Utilidade Percebida: escala de Lund (2001);

- Facilidade de Uso Percebida: escala de Lund (2001);

- Diversão Percebida: escala de Moon e Kim (2001);

- Qualidade do Sistema Percebida: escala de DeLone e McLean (1992);

- Qualidade do Conteúdo Percebida: escala de Lin e Liu (2000);

- Experiência com a Internet: Hackbarth et al. (2003);

- Nível de Preço Percebido: Liao e Cheung (2001);

- Atitude com relação à Internet Móvel: escala de Cheong e Park (2005);

- Intenção de Usar a Internet Móvel: escala de Cheong e Park (2005).

Os itens incluídos no instrumento de pesquisa foram traduzidos para o português por profissionais, com etapas de tradução e retradução para garantir que se aproximassem o máximo possível dos originais.

Foram realizados três pré-testes do questionário para avaliar a compreensão dos respondentes, eliciar melhorias e ajustar a tradução. A versão final do questionário contém 29 itens medidos via escalas Likert de cinco pontos e onze perguntas em escalas categóricas sobre o perfil demográfico dos respondentes e uso da internet e de dispositivos móveis.

\section{Amostra e Procedimentos de Coleta de Dados}

A população estudada foi a de jovens brasileiros residentes no Rio de Janeiro com ensino superior em curso. Kulviwat et al. (2007) destacam o fato de que esta "elite de jovens com acesso à tecnologia" seria um dos segmentos mais atrativos para a introdução de novas tecnologias, com seu comportamento de adoção e uso influenciando outros grupos. $\mathrm{Na}$ amostra final foram incluídos apenas os respondentes que já faziam o uso da internet por meio de celulares ou tablets e aqueles cujo perfil se aproximava do da população desejada, com idades até 25 anos, pertencentes às classes $A$ e $B$.

Todos os questionários foram preenchidos pelos próprios respondentes. Foi obtida uma amostra inicial com 454 questionários, dos quais 12 foram eliminados por apresentarem dados ausentes. Outros 137 questionários foram eliminados por não se encaixarem no perfil de interesse, sendo 25 por apresentarem 
idades acima de 25 anos, 30 por não pertencerem às classes a $A$ ou $B$ e 82 por nunca terem usado a internet móvel. Assim, a amostra final foi composta por 305 questionários válidos.

Do total de 305 respondentes, a distribuição dos gêneros foi proporcional, sendo 149 do sexo feminino (48,9\%) e 156 do sexo masculino (51,1\%). Em relação ao estado civil, todos os respondentes eram solteiros. Em relação à renda familiar média, $80,3 \%$ dos respondentes fazem parte da classe $A$ (com renda familiar acima de 10.900 reais mensais) e 19,7\% pertencem a classe $B$ (com renda familiar entre 5.450 e 10.900 reais mensais). Todos os respondentes tinham entre 17 e 25 anos de idade, sendo a média observada de 20,8, com desvio padrão de 1,76. Além disso, 98\% (299) dos respondentes afirmaram possuir um smartphone e/ou tablet, com todos os 305 afirmando já tendo acessado a internet via dispositivo móvel.

\section{RESULTADOS}

\section{Modelo de Mensuração}

Uma análise fatorial confirmatória (CFA) foi realizada para testar a validade, unidimensionalidade e confiabilidade das escalas utilizadas no modelo de mensuração.

O modelo de mensuração final, com 21 indicadores, obteve bons índices de ajuste, apresentando uma melhora significativa em relação ao modelo original. A razão x2/d.f. foi de 1,61, inferior ao valor de 3,0 sugerido por Byrne (2010). Além disso, os índices de ajuste incrementais foram maiores do que 0,90 , com um CFI (comparative fit index) de 0,95, um TLI (Tucker-Lewis index) de 0,93 e um IFI (incremental fit index) de 0,95. Por sua vez, os índices de ajuste absoluto apresentaram valores abaixo do limite de 0,08 estabelecido pela literatura (BYRNE, 2010; HAIR et al., 2009), indicando também um bom ajuste, com RMSEA (root-meansquare error of approximation) de 0,045 (C. I. de 0,035 a 0,055) e SRMR (standardized root mean-square residual) de 0,044.

A validade de face para todas as escalas utilizadas foi garantida durante o desenvolvimento do instrumento de pesquisa (escolha de escalas já utilizadas na literatura, tradução cuidadosa e pré-testes). Para verificar a validade nomológica foi analisada a matriz de correlação entre construtos, com todas as correlações sendo significativas e na direção esperada. No que diz respeito à validade convergente, foi calculada a variância extraída média para cada construto (AVE). Todos os valores de AVE estavam entre 0,68 e 0,79, evidenciando a validade convergente. Com relação à consistência interna e confiabilidade, todas as escalas utilizadas atenderam aos níveis de confiabilidade considerados adequados pela literatura (Fornell \& Larcker, 1981), apresentando valores entre 0,70 e 0,81 para o coeficiente alfa e entre 0,75 e 0,83 para a confiabilidade composta. Por fim, todas as variâncias compartilhadas foram inferiores à variância extraída pelos itens que medem os construtos, indicando validade discriminante adequada. 


\section{Modelo Estrutural}

A modelagem de equações estruturais (SEM) foi utilizada para testar o modelo proposto e as hipóteses da pesquisa. Todos os índices indicaram um bom ajuste do modelo aos dados. A razão X2/d.f. foi de 2,05, inferior ao valor de 3,0 sugerido por Byrne (2010). Além disso, os índices de ajuste incrementais foram maiores do que 0,90 , com CFI de 0,91, TLI de 0,90 e IFI de 0,91. Por sua vez, os índices de ajuste absoluto apresentaram valores abaixo do limite de 0,08 estabelecido pela literatura (HU; BENTLER, 1999; BYRNE, 2010; HAIR et al., 2009), com RMSEA de 0,06 (C. I. de 0,05 a 0,07) e SRMR de 0,076.

Uma vez que os modelos de mensuração e estrutural propostos obtiveram resultados satisfatórios, o próximo passo foi verificar as hipóteses da pesquisa. Para tanto, foi realizada uma análise da magnitude e significância dos coeficientes padronizados estimados para cada relação entre construtos (Tabela 1).

Tabela 1. Coeficientes Padronizados Estimados, Hipóteses e Significâncias

\begin{tabular}{lccc}
\hline Relação Proposta & $\begin{array}{c}\text { Coeficiente } \\
\text { Padronizado }\end{array}$ & $\begin{array}{c}\text { Hipótese } \\
\text { P-valor }\end{array}$ & Verificada \\
\hline $\mathbf{H}_{1}:$ Utilidade $\rightarrow$ Atitude & 0,62 & $<0,001$ & Sim \\
$\mathbf{H}_{2}:$ Utilidade $\rightarrow$ Intenção & 0,12 & 0,284 & Não \\
$\mathbf{H}_{3}:$ Facilidade de Uso $\rightarrow$ Atitude & 0,17 & 0,006 & Sim \\
$\mathbf{H}_{4}:$ Facilidade de Uso $\rightarrow$ Utilidade & 0,21 & 0,012 & Sim \\
$\mathbf{H}_{5}:$ Atitude $\rightarrow$ Intenção & 0,67 & $<0,001$ & Sim \\
$\mathbf{H}_{6}:$ Facilidade de Uso $\rightarrow$ Diversão & 0,13 & 0,086 & Não \\
$\mathbf{H}_{7}:$ Diversão $\rightarrow$ Atitude & 0,28 & $<0,001$ & Sim \\
$\mathbf{H}_{8}:$ Diversão $\rightarrow$ Intenção & 0,11 & 0,164 & Não \\
$\mathbf{H}_{9}:$ Experiência com Internet $\rightarrow$ Facilidade de Uso & 0,28 & $<0,001$ & Sim \\
$\mathbf{H}_{10}:$ Experiência com Internet $\rightarrow$ Diversão & 0,30 & $<0,001$ & Sim \\
$\mathbf{H}_{11}:$ Nível de Preço $\rightarrow$ Atitude & 0,07 & 0,234 & Não \\
$\mathbf{H}_{12}:$ Nível de Preço $\rightarrow$ Intenção & 0,11 & 0,057 & Não \\
$\mathbf{H}_{13}:$ Qualidade do Sistema $\rightarrow$ Utilidade & $-0,22$ & 0,013 & Não \\
$\mathbf{H}_{14}:$ Qualidade do Sistema $\rightarrow$ Facilidade de Uso & 0,43 & $<0,001$ & Sim \\
$\mathbf{H}_{15}:$ Qualidade do Conteúdo $\rightarrow$ Utilidade & 0,58 & $<0,001$ & Sim \\
$\mathbf{H}_{16}:$ Qualidade do Conteúdo $\rightarrow$ Diversão & 0,54 & $<0,001$ & Sim \\
\hline
\end{tabular}

Fonte: dados da pesquisa.

Dado os resultados ilustrados na Tabela 1, observa-se que das 16 hipóteses de pesquisa formuladas, 10 foram verificadas, sendo 8 com significância abaixo de 0,001 .

As hipóteses $\mathrm{H} 1$ até $\mathrm{H} 5$ examinam a relação entre os construtos base do Modelo de Aceitação de Tecnologia (TAM). Primeiramente, foi observado que o construto intenção de uso é predominante influenciado pelo construto atitude em relação à adoção. Estudos prévios já haviam confirmado a forte relação entre atitude e intenção de uso, além da função da atitude como mediadora entre as crenças/percepções e a intenção de uso. Os resultados indicam que a intenção de uso não é influenciada diretamente pelos construtos diversão percebida $(\mathrm{H} 8)$ e nível de preço percebido $(\mathrm{H} 12)$, mas que a atitude media essas relações. 
No caso das hipóteses $\mathrm{H} 6$ e H8, referentes à diversão percebida, foi verificado apenas o efeito direto da diversão percebida sobre a atitude, com coeficiente padronizado de 0,28 e significância no nível de 0,001. A facilidade de uso não teve efeito significativo sobre a diversão percebida, que por sua vez não influenciou diretamente a intenção de uso. Ainda sim, esse resultado demonstra a importância do indicador entretenimento no modelo TAM como antecedente relevante da atitude (MOON; KIM, 2001; CHEONG; PARK, 2005; NYSVEEN et al., 2005; WANG; WANG, 2012). Ressalta-se que Wang e Wang (2010) também não encontraram relação entre diversão e intenção de uso da internet móvel.

$\mathrm{O}$ construto experiência com a internet foi investigado nas hipóteses $\mathrm{H} 9$ e $\mathrm{H} 10$, e os resultados mostraram que exerce efeito sobre a facilidade de uso (coeficiente padronizado de $0,28, p$-valor $<0,001$ ) e sobre a diversão (coeficiente padronizado de 0,30 , p-valor $<0,001$ ). Essa relação é consistente com os resultados anteriores (LIAO; CHEUNG, 2001; HACKBARTH et al., 2003; AL-QEISI et al., 2014; HEW et al. 2015), que apontam que a experiência prévia com tecnologias similares ajuda a diminuir preocupações e estresses envolvidos na experimentação de uma nova tecnologia.

De acordo com o teste das hipóteses $\mathrm{H} 11$ e H12, foi verificado que o nível de preço percebido não possui nenhum efeito sobre a atitude e a intenção de uso da internet móvel. Esse resultado é diferente dos encontrados nos estudos desenvolvidos por Cheong e Park (2005), Wang e Wang (2010) e Gerpott e Thomas (2015). No entanto, Hew et al. (2015) também não encontraram relação significativa entre o preço pago pela assinatura e a intenção de uso da internet móvel.

As hipóteses $\mathrm{H} 13$ e $\mathrm{H} 14$ avaliaram os efeitos do construto qualidade do sistema sobre a utilidade e facilidade de uso. Ambas as relações foram consideradas significativas, assim como em outros estudos (LIN; CHEN, 2012; AL-QEISI et al., 2014). Porém, a percepção da qualidade do sistema apresenta um efeito negativo sobre o construto utilidade percebida, e tal efeito, apesar de ter uma magnitude pequena $(-0,22)$, é contrário aos resultados esperados. No entanto, já foi observada anteriormente a ausência de relação entre esses construtos. Portanto, a H13 não foi comprovada.

Por fim, avaliando as hipóteses $\mathrm{H} 15$ e H16, foi observado que, similar aos achados de Cheong e Park (2005) e Lin e Chen (2012), a qualidade do conteúdo exerce um efeito positivo e significativo sobre a utilidade e diversão, com coeficientes padronizados de 0,58 e 0,54. Esse resultado indica que a qualidade do conteúdo exerce um efeito maior sobre a utilidade do que a facilidade de uso ou a qualidade do sistema. Além disso, a diversão também é influenciada mais fortemente pela qualidade do conteúdo do que pela facilidade de uso ou a experiência com a internet. É possível conceber que a qualidade do conteúdo possui um efeito mais relevante do que a qualidade do sistema com relação às percepções de utilidade da internet móvel, que por sua vez, irá desempenhar uma função importante em desenvolver atitudes positivas em relação à adoção e utilização da internet móvel. 


\section{DISCUSSÃO DOS RESULTADOS}

O modelo estudado apresenta uma série de relações importantes para a compreensão do comportamento do consumidor diante da aceitação da internet móvel.

Primeiramente, foi observado que o único efeito significativo sobre a intenção de uso foi o da atitude em relação à adoção. Desta forma, acredita-se que a atitude de fato exerça efeito mediador entre os demais construtos e a intenção de adoção, e em alguns casos, como em relação com a diversão, essa mediação seja total.

Dentre os construtos que influenciam a atitude em relação à adoção, a utilidade teve o efeito positivo mais significativo, com o maior coeficiente entre as relações estudadas, 0,62. Portanto, é imprescindível que o consumidor perceba o uso da internet móvel como sendo efetivamente útil no dia-a-dia. Além disso, a diversão, seguida da facilidade de uso, também apresentaram efeitos diretos, positivos e significativos sobre a atitude com relação à adoção, apesar de menos pronunciados. Isto mostra que quanto mais divertida a nova tecnologia for percebida e quanto mais facilmente ela puder ser utilizada, mais positiva será a atitude do consumidor em relação à sua adoção.

A relação entre o nível de preço percebido tanto para formação da atitude quanto da intenção de uso não foi verificada. Esse resultado é diferente dos encontrados em outros estudos, em que o preço apresenta um impacto significativo e negativo na atitude e intenção de uso (LIAO; CHEUNG, 2001; WANG; WANG, 2010; GERPOTT; THOMAS, 2015). Entretanto, Hew et al. (2015) também não encontraram relação significativa entre o preço pago pela assinatura e a intenção de uso. Uma possível razão para esse resultado seria percepção da amostra selecionada. Em ambos os casos em que a relação encontrada não foi significativa a amostra é majoritariamente jovem (entre 19 e 25 anos) e de classe alta. Tendo estas características em vista, é possível que o preço não seja um fator determinante para adoção da internet móvel entre jovens universitários de classe alta. Primeiro, o valor monetário pode não ser considerado alto o suficiente para ser considerado um contraponto dos benefícios. Em segundo, é muito comum os pais arcarem com as despesas dos filhos universitários, prejudicando a avaliação por parte do grupo estudado.

A qualidade do conteúdo é significativa e determinante tanto para a utilidade quanto para a diversão. Assim, um conteúdo relevante pode fazer com que a internet móvel pareça mais agradável e divertida, contribuindo para sua adoção.

A qualidade do sistema foi significativa apenas em explicar a facilidade de uso, não apresentando relação direta com a utilidade. Isto mostra que quanto melhor for o sistema de acesso à internet por meio de dispositivos móveis, mais fácil será o seu uso.

Por fim, a experiência com a internet possui influência sobre a facilidade de uso e a diversão. Esse resultado é consistente com estudos anteriores (LIAO; CHEUNG, 2001; HACKBARTH et al., 2003; AL-QEISI et al., 2014; HEW et al. 2015). À medida que o consumidor já faz uso da Internet no computador isso irá contribuir para uma maior percepção de facilidade de uso e diversão ao utilizar a internet através de dispositivos móveis. Ou seja, quanto menos estresse e preocupação o usuário enfrentar quando utilizando um novo serviço, maior a sua aceitação do mesmo. 


\section{CONCLUSÕES}

O presente estudo contribui para melhorar a compreensão do comportamento da intenção de uso do consumidor em relação à internet móvel. Os resultados e relações verificadas neste estudo representam uma contribuição relevante para o aprofundamento e generalização do modelo de Cheong e Park (2005) e de outras extensões do Modelo de Aceitação de Tecnologia (TAM).

Dentre as principais questões abordadas nesse modelo, destaca-se a atitude em relação à internet móvel, que confirmou ser o atributo com o maior poder explicativo em relação à intenção de uso. Os resultados indicam que a utilidade e a diversão percebidas exercem uma influência positiva indireta na intenção de uso, mediada pela atitude em relação à internet móvel. De fato, à medida que a internet está cada vez mais presente na rotina diária, a importância do componente entretenimento se torna maior na definição de atitudes em relação às tecnologias análogas. No entanto, a utilidade mostrou ser mais relevante do que facilidade de uso ou a diversão percebida para formar a atitude em relação à internet móvel.

Além disso, outro resultado relevante indica que a percepção da qualidade do conteúdo possui uma função importante na formação da percepção de diversão e utilidade. Notadamente, a qualidade do conteúdo possui maior influência do que a qualidade do sistema sobre as percepções de utilidade e do que a experiência anterior com a internet sobre as percepções de facilidade de uso. No entanto, a qualidade do sistema também foi considerada significativa na formação da percepção de facilidade de uso.

No que diz respeito a implicações gerenciais, o estudo apresenta diversos achados que podem ser considerados úteis para as operadoras de telefonia móvel e também para empresas que investem neste setor. Por meio desta extensão do Modelo de Aceitação de Tecnologia (TAM) é possível entender o conjunto de percepções que determinam a predisposição dos consumidores em adotar serviços de internet móvel.

Os resultados da pesquisa indicam que a formação da intenção de uso da internet móvel está fortemente relacionada com a sua atitude. De acordo com os resultados, a atitude está fundamentada em grande parte no quão útil, divertido e fácil de usar é o serviço de internet móvel. Dentro deste contexto, a qualidade do conteúdo foi identificada como principal influência no desenvolvimento das percepções de utilidade e diversão. Dessa forma, empresas devem pensar em desenvolver serviços e produtos que disponibilizem conteúdo de interesse dos usuários, o que estimularia a adoção e uso da internet em dispositivos móveis. Além disso, um site deve ter uma visualização adequada para acessos via dispositivos móveis, oferecendo ao mesmo tempo estabilidade e velocidade adequadas para que o cliente possa usufruir dos serviços disponibilizados sem dificuldade.

Outro ponto importante é a influência que a experiência com a internet pode ter sobre a diversão e a facilidade de uso. Dado esse fato, empresas podem investir em estratégias de divulgação com interfaces similares as utilizadas na internet convencional, de forma que os clientes consigam identificar e reconhecer a compatibilidade dos serviços oferecidos (ROGERS, 2003). 


\section{LIMITAÇÕES E PESQUISAS FUTURAS}

O presente estudo foi realizado com uma amostra de conveniência, o que dificulta a generalização dos resultados. Além disso, a amostra foi composta de jovens estudantes universitários da cidade do Rio de Janeiro da classe A e B, que possuem mais acesso a tecnologias de ponta do que a população em geral. Portanto, para pesquisas futuras, sugere-se que sejam amostrados respondentes pertencentes a outras populações, ampliando a representatividade da pesquisa.

Pesquisas futuras poderiam também realizar a aplicação deste modelo de forma periódica com o objetivo de construir estudos longitudinais, permitindo assim a análise das mudanças na percepção dos clientes sobre as diversas dimensões ao longo do tempo. Adicionalmente, outras relações importantes poderiam ser investigadas com base no modelo estudado. Seria interessante averiguar as relações entre a qualidade do sistema e a diversão percebida, entre a experiência com a internet e a utilidade percebida, e entre a qualidade do conteúdo e a facilidade de uso.

Além do modelo proposto, outros recortes poderiam ser realizados sobre o tema, como, por exemplo, diferenças entre adotantes e não adotantes, ou até mesmo entre diferentes países. Dessa forma, seria possível identificar diferenças nas percepções de cada grupo.

\section{REFERÊNCIAS}

AGARWAL, R.; KARAHANNA, E. Time flies when you're having fun: cognitive absorption and beliefs about information technology usage. MIS Quarterly, v.24, n.4, pp.665-694, 2000.

AHN, T.; RYU, S.; HAN, I. The impact of Web quality and playfulness on user acceptance of online retailing. Information Management, v.44, n.3, pp.263-275, 2007.

AKTURAN, U.; TEZCAN, N. Mobile banking adoption of the youth market: Perceptions and intentions. Marketing Intelligence \& Planning, v.30, n.4, pp.444-459, 2012.

AL-QEISI, K.; DENNIS, C.; ALAMANOS, E.; JAYAWARDHENA, C. (2014). Website design quality and usage behavior: unified theory of acceptance and use of technology. Journal of Business Research, v.67, n.11, pp.2282-2290, 2014.

ANATEL. Acessos - $\quad$ Telefonia Móvel. Fev. 2015. Disponível em:< http://ftp.anatel.gov.br/dados/Acessos/Movel_Pessoal/Por_Tecnologia/csv/>. Acesso em: 05 ago. 2015.

BRUNER, G. C.; KUMAR, A. Explaining consumer acceptance of handheld Internet devices. Journal of Business Research, v.58, n.5, pp.553-558, 2005.

BYRNE, B. M. Structural Equation Modeling with AMOS: Basic Concepts, Applications and Programming. 2nd ed. Routledge, NY, 2010.

CHEN, Y. H.; CORKINDALE, D. Towards an understanding of behavioral intention to use online news services - An exploratory study. Internet Research, v.18, n.3, pp.286-312, 2008.

CHEONG, J. H.; PARK, M. C. Mobile internet acceptance in Korea. Internet Research, v.15, n.2, pp.125-140, 2005.

CHEUNG, R.; VOGEL, D. Predicting user acceptance of collaborative technologies: An extension of the technology acceptance model for e-learning. Computers \& Education, v.63, pp.160-175, 2013.

CHILDERS, T.; CARR, C.; PECK, J.; CARSON, S. Hedonic and utilitarian motivations for online retail shopping behavior. Journal of Retailing, v.77, n.4, pp.511-35, 2001

ÇELIK, H. Influence of social norms, perceived playfulness and online shopping anxiety on customers' adoption of online retail shopping. International Journal of Retail \& Distribution Management, v.39, n.6, pp.390-413, 2011.

CHUNG, J.; TAN, F.B. Antecedents of perceived playfulness: An exploratory study on user acceptance of general information-searching websites. Information \& Management, v.41, n.7, pp.869-881, 2004.

DAVIS, F. D. Perceived Usefulness, Perceived Ease of Use, and User Acceptance of Information Technology. MIS Quarterly, v.13, n.3, pp,319-339, 1989.

DAVIS, F. D.; BAGOZZI, R. P.; WARSHAW, P. R. User Acceptance of Computer Technology: A Comparison of Two Theoretical Models. Management Science, 35(8), 982-1002, 1989. 
DELONE, W.; MCLEAN, E. Information systems success: the quest for the dependent variable, Information Systems Research, v.3, n.1, pp.60-95, 1992.

DELONE, W.; MCLEAN, E. The DeLone and McLean model of information system: A ten year update. Journal of Management Information Systems, 19(4), 9-30, 2003.

E-BIT. Webshoppers 2015 31a edição. 2015. Disponível em: < http://www.ebitempresa.com.br/web-shoppers.asp>. Acesso em: 24 jul. 2015.

FANG, X. W.; CHAN, S.; BRZEZINSKI, J.; XU, S. Moderating effects of task types on wireless technology acceptance. Journal of Management Information Systems, v.22, pp.123-157, 2006.

FERREIRA, J. B.; ROCHA, A.; SILVA, J. F. Impacts of technology readiness on emotions and cognition in Brazil. Journal of Business Research, v.67, n.5, pp.865-873, 2014.

FISHBEIN, M.; AJZEN, I. Belief, Attitude. Intention and Behavior: An Introduction to Theory and Research. Reading, MA: Addison-Wesley Publishing, June 1975.

FORNELL, C.; LARCKER, D. F. Evaluating Structural Equation Models with Unobservable Variables and Measurement Error. Journal of Marketing Research, v.18, pp.39-50, 1981.

GARTNER. Top 10 strategic predictions for 2015 and beyond: Digital business is driving 'big change'. Research. 04 oct. 2014. 2014. Disponível em: <https://www.gartner.com/doc/2864817?refval=\&pcp=mpe>. Acesso em: 24 jul. 2015.

GEFEN, D.; KARAHANNA, E.; STRAUB, D. W. Trust and TAM in online shopping: an integrated model. MIS quarterly, v.27, n.1, pp.51-90, 2003.

GENTRY, L.; CALANTONE, R. A comparison of three models to explain shop-bot use on the web. Psychology \& Marketing, v.19, pp.945-956, 2002.

GERPOTT, T. J. Attribute perceptions as factors explaining Mobile Internet acceptance of cellular customers in Germany - An empirical study comparing actual and potential adopters with distinct categories of access appliances. Expert Systems with Applications, v.38, pp.2148-2162, 2011.

GERPOTT, T. J.; THOMAS, S. Empirical research on mobile internet usage: A meta-analysis of the literature. Telecommunications Policy, v.38, n.3, pp.291-310, 2014.

HACKBARTH, G.; GROVER, V.; YI, M. Y. Computer playfulness and anxiety: positive and negative mediators of the system experience effect on perceived ease of use. Information and Management, v.40, n.3, pp.221-232, 2003.

HAILIN, M. S. Mobile Internet Experience Research Based on TAM, E-Product E-Service and E-Entertainment, ICEEE International Conference, p.1-4, 2010.

HAIR, J. F.; BLACK, W. C.; BABIN, B. J.; ANDERSON, R. E. Multivariate Data Analysis. 7. ed. Upper Saddle River: Prentice-Hall, 2009.

HEIJDEN, H. Factors influencing the usage of Websites: the case of generic portal in The Netherlands. Information \& Management, v.40, n.6, pp.541-549, 2003.

HEW, J.; LEE, V.; OOI, K.; WEI, J. What catalyses mobile apps usage intention: an empirical analysis. Industrial Management \& Data Systems, v.115, n.7, pp.1269-1291, 2015.

HU, L.; BENTLER, P. M. Cutoff criteria for fit indexes in covariance structure analysis: Conventional criteria versus new alternatives. Structural Equation Modeling, v.6, n.1, pp.1-55, 1999.

INTERNET SOCIETY. Global Internet Report 2015: Mobile evolution and development of the internet. 2015. Disponível em: <http://www.internetsociety.org/globalinternetreport/assets/download/IS_web.pdf>. Acesso em: 24 jul. 2015.

INTERNATIONAL TELECOMMUNICATIONS UNION. ICT Facts \& Figures. 2015. Disponível em: < http://www.itu.int/en/ITU-D/Statistics/Documents/facts/ICTFactsFigures2015.pdf>. Acesso em: 24 jul. 2015.

KIM, D. J.; HWANG, Y. A study of mobile internet user's service quality perceptions from a user's utilitarian and hedonic value tendency perspectives. Information Systems Frontiers, v.14, n.2, pp.409-421, 2012.

KULVIWAT, S.; BRUNER II, G. C.; KUMAR, A.; NASCO, S. A.; CLARK, T. Toward a Unified Theory of Consumer Acceptance Technology. Psychology and Marketing, v.24, n.12, pp.1059-1084, 2007.

LAROSE, R.; GREGG, J. L.; STROVER, S.; STRAUBHAAR, J.; CARPENTER, S. Closing the rural broadband gap: Promoting adoption of the internet in rural America. Telecommunications Policy, v.31, n.6, pp.359-373, 2007.

LIAO, Z.; CHEUNG, M. T. Internet-based e-shopping and consumer attitudes: an empirical study, Information and Management, v.38, n.5, pp.299-306, 2001.

LIN, K. E-Learning continuance intention: Moderating effects of e-learning experience. Computers \& Education, v.56, pp.515-526, 2012.

LIN, J. C.; LU, H. Towards an understanding the behavioral intention to use a web site, International Journal of Information Management, v.20, n.3, pp.197-208, 2000. 
LIN, T.; CHEN, C. Validating the satisfaction and continuance of e-learning systems: Combining TAM and IS success models. International Journal of Distance Education Technologies, v.10, n.1, pp.44-54, 2012.

LU, H.; SU, Y. S. Factors affecting purchase intention on mobile shopping Web sites. Internet Research, v.19, n.4, pp.442458, 2009.

LUND, A. M. Measuring usability with the USE questionnaire. Usability Interface, v.8, n.2, pp.3-6, 2001.

MONROE, K. B. Price and Customers' Perceptions of Value. Visionary Pricing: Reflections and Advances in Honor of Dan Nimer (Advances in Business Marketing and Purchasing, Volume 19. Emerald Group Publishing Limited, pp.129-152, 2012.

MOON, J. W.; KIM, Y. G. Extending the TAM for a World-Wide-Web context. Information and Management, v.38, n.4, pp.217-230, 2001.

MORGAN STANLEY. Internet $2010 . \quad$ Trends. Disponível em: <http://www.morganstanley.com/institutional/techresearch/pdfs/Internet_Trends_041210.pdf>. Acesso em: 12 out. 2011.

NASCO, S. N.; KULVIWAT, S.; KUMAR, A.; BRUNER II, G. C. The CAT model: Extensions and Moderators of Dominance in Technology Acceptance. Psychology and Marketing, v.25, n.10, pp.987-1005, 2008.

NASERI, M. B.; ELLIOT, G. Role of demographics, social connectedness and prior internet experience in adoption of online shopping: Applications for direct marketing. Journal of Targeting, Measurement and Analysis for Marketing, v.19, n.2, pp.69-84, 2011.

NASRI, W.; CHARFEDDINE, L. Factors affecting the adoption of internet banking in Tunisia: An integration theory of acceptance model and theory of planned behavior. Journal of High Technology Management Research, v.23, n.1, pp.1$14,2012$.

NYSVEEN, H.; PEDERSEN, P. E.; THORBJORNSEN, H. Intentions to use mobile services: Antecedents and crossservice comparisons. Journal of the Academy of Marketing Science, v.33, n.3, pp.330-346, 2005.

OH, S. H., KIM, Y. M., LEE, C. W., SHIM, G. Y., PARK, M. S., \& JUNG, H. S. Consumer Adoption of Virtual Stores in Korea: Focusing on the Role of Trust and Playfulness. Psychology \& Marketing, v.26, n.7, pp.652-668, 2009.

PAGANI, M. Determinants of adoption of third generation mobile multimedia services. Journal of International Marketing, V.18, n.3, pp.46-59, 2004

PAI, F., \& HUANG, K. Applying the Technology Acceptance Model to the introduction of healthcare information systems. Technological Forecasting and Social Change, v.78, n.4, pp.650-660, 2011.

PARASURAMAN, A.; GREWAL, D.; KRISHNAN, R. Marketing Research. 2. ed. South Western College Pub, 2006.

ROBEY, D. User Attitudes and Management Information System use. Academy of Management Journal, v.22, n.3, pp.527538, 1979.

ROGERS, E. Diffusion of Innovations. 5 ed. New York: Free Press, 2003.

SCHREIBER, J. B.; STAGE, F. K.; KING, J.; NORA, A.; BARLOW, E. A. Reporting Structural Equation Modeling and Confirmatory Factor Analysis Results: A Review. Journal of Educational Research, v.99, n.6, pp.323-337, 2006.

SCHULTZ, R. L.; SLEVIN, D. P. Implementing Operations Research/Management Science. New York: American Elsevier, pp.153-182, 1975.

SHEPPARD, B. H.; HARTWICK, J.; WARSHAW, P. R. The Theory of Reasoned Action: A Meta-Analysis of Past Research with Recommendations for Modifications and Future Research. Journal of Consumer Research, v.15, n.3, pp.325-343, 1988.

TAN, F. B.; CHOU, J. P. C. The Relationship Between Mobile Service Quality, Perceived Technology Compatibility, and Users' Perceived Playfulness in the Context of Mobile Information and Entertainment Services. International Journal of Human-Computer Interaction, v.24, n.7, pp.649-671, 2008.

TEO, T. S. H.; LIM, V. K. G.; LAI, R. Y. C. Intrinsic and extrinsic motivation in Internet usage. International Journal of Management Science, v.27, n.1, pp.25-37, 1999.

TSAI, H. S.; LAROSE, R. Broadband internet adoption and utilization in the inner city: A comparison of competing theories. Computers in Human Behavior, v.51, pp.344-355, 2015.

TSENG, F.; TENG, C. Antecedents for user intention to adopt another auction site. Internet Research, v.24, n.2, pp.205222, 2014.

VAN DEURSEN, A. J. A. M.; VAN DIJK, J. A. G. M.; PETERS, O. Rethinking Internet skills: the contribution of gender, age, education, Internet experience, and hours online to medium-and content-related Internet skills. Poetics, v.39, n.2, pp.125-144, 2011.

VENKATESH, V. Creation of favorable user perceptions: exploring the role of intrinsic motivation. MIS Quarterly, v.23, n.2, pp.239-260, 1999. 
VENKATESH, V.; DAVIS, F. D. A model of the antecedents of perceived ease of use: development and text. Decision Sciences, v.27, n.3, pp.41-481, 2000.

WANG, Y. S.; LIN, H. H.; LUARN, P. Predicting consumer intention to use mobile service. Information Systems Journal, v.16, n.2, pp.157-179, 2006.

WANG, K.; LIN, C. The adoption of mobile value-added services. Managing Service Quality: An International Journal, v.22, n.2, pp.184-208, 2012.

WANG, H.; WANG, S. User acceptance of mobile internet based on the unified theory of acceptance and use of technology: investigating the determinants and gender differences. Social Behavior and Personality, v.38, n.3, pp.415-426, 2010.

WEBSTER, J.; MARTOCCHIO, J. J. Microcomputer playfulness: development of a measure with workplace implications. MIS Quarterly, v.16, n.2, pp.201-226, 1992.

YOON, S.; OH, S.; SONG, S.; KIM, K. K.; KIM, Y. Higher quality or lower price? How value-increasing promotions affect retailer reputation via perceived value. Journal of Business Research, v.67, n.10, pp.2088-2096, 2014.

YOUSAFZAI, S. Y.; FOXALL, G. R.; PALLISTER, J. G. Technology Acceptance: a Meta-Analysis of the TAM: Part 1. Journal of Modeling in Management, v.2, n.3, pp.251-280, 2007.

ZEITHAML V. A. Consumer Perceptions of Price, Quality and Value: A Mean-end Model and Synthesis of Evidence. Journal of Marketing, v.52, n.3, pp.2-22, 1988. 